\title{
REAL DIVISORS ALGEBRAICALLY EQUIVALENT TO ZERO
}

\author{
W. KuCHARZ
}

\section{Introduction}

Let $X$ be a smooth projective variety over $\mathbb{R}$ of dimension $n$ (that is, an integral smooth projective scheme over $\mathbb{R}$ of dimension $n$ ). We endow the set $X(\mathbb{R})$ of $\mathbb{R}$-rational points of $X$ with the topology induced by the usual metric topology on $\mathbb{R}$ and assume that $X(\mathbb{R})$ is nonempty. Thus $X(\mathbb{R})$ is a closed $n$-dimensional manifold of class $\mathcal{C}^{\infty}$. We have the cycle homomorphism

$$
c \ell_{\mathbb{R}}: Z^{k}(X) \rightarrow H^{k}(X(\mathbb{R}), \mathbb{Z} / 2)
$$

defined on the group $Z^{k}(X)$ of algebraic cycles on $X$ of codimension $k$ : for any closed subvariety $V$ of $X$ of codimension $k$, the cohomology class $c \ell_{\mathbb{R}}(V)$ is Poincaré dual to the homology class in $H_{n-k}(X(\mathbb{R}), \mathbb{Z} / 2)$ represented by $V(\mathbb{R})$ if $\operatorname{dim} V(\mathbb{R})=n-k$ (cf. [4] or [2, Section 11.3] for the definition of this homology class) and $c \ell_{\mathbb{R}}(V)=0$ if $\operatorname{dim} V(\mathbb{R})<n-k$. Of fundamental interest in real algebraic geometry is the image

$$
H_{\mathrm{alg}}^{k}(X(\mathbb{R}), \mathbb{Z} / 2)=c \ell_{\mathbb{R}}\left(Z^{k}(X)\right)
$$

of $Z^{k}(X)$ via $c \ell_{\mathbb{R}}$ (cf. [3] for a short survey of its properties and applications). Recently also the subgroup

$$
\operatorname{Alg}^{k}(X(\mathbb{R}))=c \ell_{\mathbb{R}}\left(Z_{\mathrm{alg}}^{k}(X)\right)
$$

of $H_{\mathrm{alg}}^{k}(X(\mathbb{R}), \mathbb{Z} / 2)$, where $Z_{\mathrm{alg}}^{k}(X)$ is the subgroup of $Z^{k}(X)$ consisting of all cycles algebraically equivalent to 0 , proved to be very useful (cf. [7, Chapter 10] for the theory of algebraic equivalence). The group $\operatorname{Alg}^{k}(X(\mathbb{R}))$ is often highly nontrivial, as illustrated for example by Theorem 1.3 below, dealing with $\operatorname{Alg}^{1}(X(\mathbb{R}))$. The behavior of $H_{\mathrm{alg}}^{n-k}(X(\mathbb{R}), \mathbb{Z} / 2)$ is strongly influenced by $\operatorname{Alg}^{k}(X(\mathbb{R}))$, cf. $[1,12]$. Some crucial constructions of [14], at the borderline between real algebraic geometry and differential topology, depend on $\mathrm{Alg}^{k}(-)$. In [13] it is proved, among other things, that $\operatorname{Alg}^{1}(X(\mathbb{R}))$ is a birational invariant of $X$. In general, it is very difficult to compute explicitly $\operatorname{Alg}^{k}(X(\mathbb{R}))$ for $0<k<n$. The present paper shows that $\operatorname{Alg}^{1}(X(\mathbb{R}))$ can be described in a purely topological way; in fact, every cohomology class in $\operatorname{Alg}^{1}(X(\mathbb{R})) \subseteq H^{1}(X(\mathbb{R}), \mathbb{Z} / 2)$ comes from a conjugation invariant cohomology class in $H^{1}(X(\mathbb{C}), \mathbb{Z})$ by restriction

Received May 31, 2003. 
to $X(\mathbb{R})$ and reduction modulo 2. In order to state our result we need some preparation.

The set $X(\mathbb{C})$ of all $\mathbb{C}$-rational points of $X$ will be regarded as a complex manifold. The Galois group $G=\operatorname{Gal}(\mathbb{C} / \mathbb{R})=\{1, \sigma\}$ of $\mathbb{C}$ over $\mathbb{R}$ acts on $X(\mathbb{C})$ and we let

$$
\sigma_{X}: X(\mathbb{C}) \rightarrow X(\mathbb{C})
$$

denote the antiholomorphic involution corresponding to $\sigma$. Moreover, $G$ acts on $H^{1}(X(\mathbb{C}), \mathbb{Z})$, the action of $\sigma$ being given by the induced isomorphism

$$
\sigma_{X}^{*}: H^{1}(X(\mathbb{C}), \mathbb{Z}) \rightarrow H^{1}(X(\mathbb{C}), \mathbb{Z}) .
$$

We have

$$
H^{2}\left(G, H^{1}(X(\mathbb{C}), \mathbb{Z})\right)=\operatorname{Ker}\left(1-\sigma_{X}^{*}\right) / \operatorname{Im}\left(1+\sigma_{X}^{*}\right),
$$

where 1 stands for the identity map of $H^{1}(X(\mathbb{C}), \mathbb{Z})$. Since in this paper we do not use properties of the group cohomology, the reader may consider the equality above as the definition of $H^{2}\left(G, H^{1}(X(\mathbb{C}), \mathbb{Z})\right)$. Note that $\operatorname{Ker}\left(1-\sigma_{X}^{*}\right)$ is equal to the subgroup $H^{1}(X(\mathbb{C}), \mathbb{Z})^{G}$ of $H^{1}(X(\mathbb{C}), \mathbb{Z})$ consisting of all elements fixed by $G$.

We identify $X(\mathbb{R})$ with the subset of $X(\mathbb{C})$ consisting of all points fixed by $G$, that is $X(\mathbb{R})=\left\{x \in X(\mathbb{C}) \mid \sigma_{X}(x)=x\right\}$. Denoting by $i_{X}: X(\mathbb{R}) \hookrightarrow X(\mathbb{C})$ the inclusion map, one readily verifies that the induced homomorphism $i_{X}^{*}$ : $H^{1}(X(\mathbb{C}), \mathbb{Z}) \rightarrow H^{1}(X(\mathbb{R}), \mathbb{Z})$ satisfies

$$
i_{X}^{*}\left(\operatorname{Im}\left(1+\sigma_{X}^{*}\right)\right) \subseteq 2 H^{1}(X(\mathbb{R}), \mathbb{Z}) .
$$

Hence we can define a homomorphism

$$
\begin{array}{r}
i_{X}^{2}: H^{2}\left(G, H^{1}(X(\mathbb{C}), \mathbb{Z})\right) \rightarrow H^{1}(X(\mathbb{R}), \mathbb{Z} / 2), \\
i_{X}^{2}\left(u+\operatorname{Im}\left(1+\sigma_{X}^{*}\right)\right)=i^{*}(u)_{2} \text { for all } u \text { in } H^{1}(X(\mathbb{C}), \mathbb{Z})^{G},
\end{array}
$$

where $i^{*}(u)_{2}$ denotes the image of $i^{*}(u)$ under the reduction modulo 2 homomorphism $H^{1}(X(\mathbb{R}), \mathbb{Z}) \rightarrow H^{1}(X(\mathbb{R}), \mathbb{Z} / 2)$.

Theorem 1.1. If $X$ is a smooth projective variety over $\mathbb{R}$ with $X(\mathbb{R})$ nonempty, then

$$
\operatorname{Alg}^{1}(X(\mathbb{R}))=i_{X}^{2}\left(H^{2}\left(G, H^{1}(X(\mathbb{C}), \mathbb{Z})\right)\right)
$$

Furthermore, $i_{X}^{2}$ is injective if $X$ is an Abelian variety over $\mathbb{R}$.

As a consequence we obtain that $\operatorname{Alg}^{1}(X(\mathbb{R}))$ is completely determined by the action of $G$ on $X(\mathbb{C})$. In particular, we have the following result.

Corollary 1.2. Let $X$ and $Y$ be smooth projective varieties over $\mathbb{R}$ with $X(\mathbb{R})$ and $Y(\mathbb{R})$ nonempty. If $X(\mathbb{C})$ and $Y(\mathbb{C})$ are $G$-equivariantly homeomorphic, then the groups $\operatorname{Alg}^{1}(X(\mathbb{R}))$ and $\operatorname{Alg}^{1}(Y(\mathbb{R}))$ are isomorphic. 
Proof. Let $h: X(\mathbb{C}) \rightarrow Y(\mathbb{C})$ be a $G$-equivariant homeomorphism, and let $h_{0}:$ $X(\mathbb{R}) \rightarrow Y(\mathbb{R})$ be the restriction of $h$. We have the following commutative diagram:

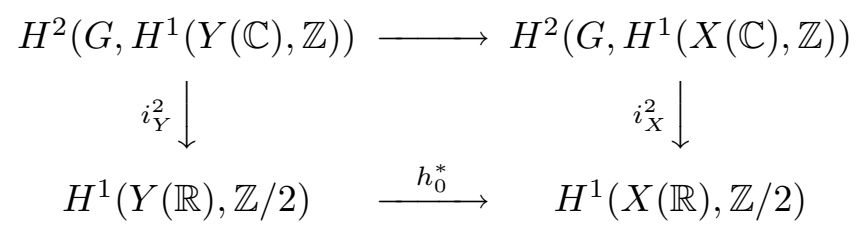

where the upper horizontal arrow is the isomorphism induced by $h^{*}: H^{1}(Y(\mathbb{C}), \mathbb{Z})$ $\rightarrow H^{1}(X(\mathbb{C}), \mathbb{Z})$. Hence, in view of Theorem 1.1, $h_{0}^{*}$ establishes an isomorphism from $\operatorname{Alg}^{1}(Y(\mathbb{R}))$ onto $\operatorname{Alg}^{1}(X(\mathbb{R}))$.

Theorem 1.1 will be proved in Section 2. Its proof depends on some classical results [6], whose modern treatment is contained in [5, 9, 11, 20].

In order to demonstrate that $\operatorname{Alg}^{1}(-)$ is an interesting invariant we shall now cite a result of [15]. The $k$ th Stiefel-Whitney class of a closed $\mathcal{C}^{\infty}$ manifold $M$ will be denoted by $w_{k}(M)$, while $[M]$ will stand for the fundamental class of $M$ in $H_{m}(M, \mathbb{Z} / 2), m=\operatorname{dim} M$. As usual we will use $\cup$ and $<,>$ to denote the cup product and scalar (Kronecker) product.

Theorem 1.3. Let $M$ be a closed $\mathcal{C}^{\infty}$ manifold of dimension $m$ with $m \geq 2$. Given a subgroup $G$ of $H^{1}(M, \mathbb{Z} / 2)$, the following conditions are equivalent:

(a) There exist a smooth projective variety $X$ over $\mathbb{R}$ and a $\mathcal{C}^{\infty}$ diffeomorphism $\varphi: X(\mathbb{R}) \rightarrow M$ such that $\varphi^{*}(G)=\operatorname{Alg}^{1}(X(\mathbb{R}))$,

(b) $G$ is contained in the image of the reduction modulo 2 homomorphism $H^{1}(M, \mathbb{Z}) \rightarrow H^{1}(M, \mathbb{Z} / 2)$, and for each integer $\ell, 1 \leq \ell \leq m$, and all $u_{1}, \ldots, u_{\ell}$ in $G$, one has

$$
<u_{1} \cup \ldots \cup u_{\ell} \cup w_{i_{1}}(M) \cup \ldots \cup w_{i_{r}}(M),[M]>=0
$$

for all nonnegative integers $i_{1}, \ldots, i_{r}$ with $i_{1}+\cdots+i_{r}=m-\ell$.

Reference for the proof. [15].

In [15] the reader also can find some results similar to Theorem 1.3 for $\mathrm{Alg}^{k}(-)$ with $k \geq 2$.

\section{Proof of Theorem 1.1}

We begin by introducing notation and terminology. As usual, if the Galois group $G=\operatorname{Gal}(\mathbb{C} / \mathbb{R})=\{1, \sigma\}$ acts on an Abelian group $M$, we denote by $M^{G}$ the subgroup of $M$ consisting of all elements fixed by $G$.

Let $V$ be a complex manifold endowed with an antiholomorphic involution $\sigma_{V}$. The group $G$ acts on the Picard group $\operatorname{Pic}(V)$ of isomorphism classes of holomorphic line bundles on $V$. If an element $\xi$ of $\operatorname{Pic}(V)=H^{1}\left(V, \mathcal{O}_{V}^{*}\right)$ is represented by a Čech cocycle $\left\{U_{i}, g_{i j}\right\}$, then $\xi^{\sigma}$ is represented by the Cech cocycle $\left\{\sigma_{V}\left(U_{i}\right), \sigma \circ g_{i j} \circ \sigma_{V}\right\}$. Note that the action of $G$ on $\operatorname{Pic}(V)$ can be restricted to 
the subgroup $\operatorname{Pic}^{0}(V)$ of $\operatorname{Pic}(V)$ consisting of the isomorphism classes of holomorphic line bundles that are topologically trivial. In particular, $\operatorname{Pic}(V)^{G}$ and $\operatorname{Pic}^{0}(V)^{G}$ are defined. We say that a holomorphic line bundle $(L, \pi, V)$ on $V$ is defined over $\mathbb{R}$ if it comes with an antiholomorphic involution $\sigma_{L}: L \rightarrow L$ such that $\pi \circ \sigma_{L}=\sigma_{V} \circ \pi$, and for each point $x$ in $V$, the restriction $\sigma_{L}: L_{x} \rightarrow L_{\sigma_{V}(x)}$ is anti-linear, that is $\sigma_{L}(z v)=\sigma(z) \sigma_{L}(v)$ for all $z$ in $\mathbb{C}$ and $v$ in $L_{x}$. Holomorphic line bundles defined over $\mathbb{R}$ (on $V$ ) form a category in which morphisms are homomorphisms of holomorphic line bundles that commute with the given involutions on the total spaces. If $L$ is defined over $\mathbb{R}$, then its class in $\operatorname{Pic}(V)$ (ignore the involution on $L$ ) belongs to $\operatorname{Pic}(V)^{G}$. We will regard $(L(\mathbb{R}), \pi(\mathbb{R}), V(\mathbb{R}))$, where

$$
L(\mathbb{R})=\left\{v \in L \mid \sigma_{L}(v)=v\right\}, \quad V(\mathbb{R})=\left\{x \in V \mid \sigma_{V}(x)=x\right\},
$$

and $\pi(\mathbb{R}): L(\mathbb{R}) \rightarrow V(\mathbb{R})$ is the restriction of $\pi: L \rightarrow V$, as a topological real line bundle on $V(\mathbb{R})$.

Now let $X$ be a smooth projective variety over $\mathbb{R}$ with $X(\mathbb{R})$ nonempty. Given an invertible sheaf $\mathcal{L}$ on $X$, we denote by $\mathcal{L}(\mathbb{R})$ the topological real line bundle on $X(\mathbb{R})$ determined by $\mathcal{L}$ in the usual way. The correspondence which assigns to $\mathcal{L}$ the first Stiefel-Whitney class $w_{1}(\mathcal{L}(\mathbb{R}))$ of $\mathcal{L}(\mathbb{R})$ gives rise to a canonical homomorphism

$$
w_{X}: \operatorname{Pic}(X) \rightarrow H^{1}(X(\mathbb{R}), \mathbb{Z} / 2),
$$

defined on the Picard group $\operatorname{Pic}(X)$ of isomorphism classes of invertible sheaves on $X$. If $\mathcal{O}(D)$ is the invertible sheaf associated with a Weil divisor $D$ on $X$, then $w_{1}(\mathcal{O}(D)(\mathbb{R}))=c \ell_{\mathbb{R}}(D)$, cf. [4, p. 498] (obviously, $Z^{1}(X)$ is the group of Weil divisors on $X)$. Hence

$$
\operatorname{Alg}^{1}(X(\mathbb{R}))=w_{X}\left(\operatorname{Pic}^{0}(X)\right),
$$

where $\operatorname{Pic}^{0}(X)$ is the subgroup of $\operatorname{Pic}(X)$ consisting of the isomorphism classes of invertible sheaves of the form $\mathcal{O}(D)$ for $D$ in $Z_{\text {alg }}^{1}(X)$, cf. Section 1.

Recall that the complex manifold $X(\mathbb{C})$ is endowed with the antiholomorphic involution $\sigma_{X}$ and $X(\mathbb{R})=\left\{x \in X(\mathbb{C}) \mid \sigma_{X}(x)=x\right\}$. In particular, $\operatorname{Pic}(X(\mathbb{C}))$ and $\operatorname{Pic}(X(\mathbb{C}))^{G}$ are defined, and it makes sense to talk about holomorphic line bundles on $X(\mathbb{C})$ defined over $\mathbb{R}$. For any invertible sheaf $\mathcal{L}$ on $X$, we let $\mathcal{L}(\mathbb{C})$ denote the associated holomorphic line bundle on $X(\mathbb{C})$. Clearly, $\mathcal{L}(\mathbb{C})$ is defined over $\mathbb{R}$ (with the natural antiholomorphic involution on the total space). Moreover, $\mathcal{L}(\mathbb{R})$ and $\mathcal{L}(\mathbb{C})(\mathbb{R})$ can be canonically identified. The assignment $\mathcal{L} \rightarrow \mathcal{L}(\mathbb{C})$ gives rise to a canonical homomorphism

$$
\iota_{X}: \operatorname{Pic}(X) \rightarrow \operatorname{Pic}(X(\mathbb{C}))^{G} .
$$

Since $X$ is projective and $X(\mathbb{R}) \neq \emptyset$, the homomorphism $\iota_{X}$ is an isomorphism, and

$$
\iota_{X}\left(\operatorname{Pic}^{0}(X)\right)=\operatorname{Pic}^{0}(X(\mathbb{C}))^{G} .
$$

Furthermore, two holomorphic line bundles on $X(\mathbb{C})$ defined over $\mathbb{R}$ represent the same element in $\operatorname{Pic}(X(\mathbb{C}))$ if and only if they are isomorphic as holomorphic line bundles defined over $\mathbb{R}$. Indeed, by the GAGA principle [19], holomorphic 
line bundles on $X(\mathbb{C})$ can be identified with invertible sheaves on the variety $X \times_{\mathbb{R}} \mathbb{C}$ over $\mathbb{C}$. A holomorphic line bundle $L$ on $X(\mathbb{C})$ is topologically trivial if and only if it corresponds to a divisor on $X \times_{\mathbb{R}} \mathbb{C}$ that is algebraically equivalent to 0 , cf. [8]. Therefore the assertions above follow from descent theory [10]. Consequently, the composed homomorphism

$$
w_{X}^{G}=w_{X} \circ \iota_{X}^{-1}: \operatorname{Pic}(X(\mathbb{C}))^{G} \rightarrow H^{1}(X(\mathbb{R}), \mathbb{Z} / 2)
$$

can be described as follows: given $\xi$ in $\operatorname{Pic}(X(\mathbb{C}))^{G}$, choose a holomorphic line bundle $L$ on $X(\mathbb{C})$ defined over $\mathbb{R}$ and representing $\xi$, and then

$$
w_{X}^{G}(\xi)=w_{1}(L(\mathbb{R}))
$$

In view of $(2.1)$, we get

$$
w_{X}^{G}\left(\operatorname{Pic}^{0}(X(\mathbb{C}))^{G}\right)=\operatorname{Alg}^{1}(X(\mathbb{R})) .
$$

Formulas (2.2) and (2.3) will be starting points in the proof of Theorem 1.1.

It will be convenient to adopt the following convention. Let $W$ be a topological space and let $U$ be a subspace of $W$. For $\Pi=\mathbb{Z}$ or $\Pi=\mathbb{Z} / 2$, the homomorphism $H^{1}(W, \Pi) \rightarrow H^{1}(U, \Pi)$, induced by the inclusion map $U \hookrightarrow W$, will be called the restriction homomorphism. The homomorphism $H^{1}(W, \mathbb{Z}) \rightarrow H^{1}(U, \mathbb{Z} / 2)$, which is the composite of the restriction homomorphism $H^{1}(W, \mathbb{Z}) \rightarrow H^{1}(U, \mathbb{Z})$ and the reduction modulo 2 homomorphism $H^{1}(U, \mathbb{Z}) \rightarrow H^{1}(U, \mathbb{Z} / 2)$, also will be called the restriction homomorphism.

Let $A$ be an Abelian variety over $\mathbb{R}$. We regard $A(\mathbb{R})$ as a real Lie group. For any point $x$ in $A(\mathbb{R})$, let $t_{x}: A \rightarrow A$ denote translation by $x$. The induced map $t_{x}(\mathbb{R}): A(\mathbb{R}) \rightarrow A(\mathbb{R})$ is translation by $x$ on $A(\mathbb{R})$. Observe that

$$
H^{1}(A(\mathbb{R}), \mathbb{Z} / 2)^{\mathrm{inv}}=\left\{\xi \in H^{1}(A(\mathbb{R}), \mathbb{Z} / 2) \mid t_{x}(\mathbb{R})^{*}(\xi)=\xi \text { for all } x \in A(\mathbb{R})\right\}
$$

is a subgroup of $H^{1}(A(\mathbb{R}), \mathbb{Z} / 2)$.

Lemma 2.4. With notation as above,

$$
\operatorname{Alg}^{1}(A(\mathbb{R})) \subseteq H^{1}(A(\mathbb{R}), \mathbb{Z} / 2)^{\text {inv }}
$$

Proof. Let $x$ be a point in $A(\mathbb{R})$. If $\mathcal{L}$ is an invertible sheaf on $A$ whose isomorphism class is in $\operatorname{Pic}^{0}(A)$, then the sheaves $t_{x}^{*} \mathcal{L}$ and $\mathcal{L}$ are isomorphic, cf. $\left[17\right.$, Proposition 9.2] and hence $w_{1}\left(\left(t_{x}^{*} \mathcal{L}\right)(\mathbb{R})\right)=w_{1}(\mathcal{L}(\mathbb{R}))$. Since $\left(t_{x}^{*} \mathcal{L}\right)(\mathbb{R})$ is isomorphic to $t_{x}(\mathbb{R})^{*} \mathcal{L}(\mathbb{R})$, we complete the proof by applying $(2.1)$.

Denote by $A(\mathbb{R})_{0}$ the connected component of $A(\mathbb{R})$ containing the identity element of the group $A(\mathbb{R})$. Let

$$
\begin{aligned}
R_{A} & : H^{1}(A(\mathbb{C}), \mathbb{Z}) \rightarrow H^{1}(A(\mathbb{R}), \mathbb{Z} / 2) \\
r_{A} & : H^{1}(A(\mathbb{C}), \mathbb{Z}) \rightarrow H^{1}\left(A(\mathbb{R})_{0}, \mathbb{Z} / 2\right) \\
\rho_{A} & : H^{1}(A(\mathbb{R}), \mathbb{Z} / 2) \rightarrow H^{1}\left(A(\mathbb{R})_{0}, \mathbb{Z} / 2\right)
\end{aligned}
$$

be the restriction homomorphisms, and let

$$
\rho_{A}^{\text {inv }}: H^{1}(A(\mathbb{R}), \mathbb{Z} / 2)^{\text {inv }} \rightarrow H^{1}\left(A(\mathbb{R})_{0}, \mathbb{Z} / 2\right)
$$


be the restriction of $\rho_{A}$.

Lemma 2.5. With notation as above,

$$
R_{A}\left(H^{1}(A(\mathbb{C}), \mathbb{Z})\right)=H^{1}(A(\mathbb{R}), \mathbb{Z} / 2)^{\text {inv }},
$$

and $\rho_{A}^{\text {inv }}$ is an isomorphism.

Proof. First we show that $\rho_{A}^{\text {inv }}$ is injective. Suppose that $\xi$ is in $H^{1}(A(\mathbb{R}), \mathbb{Z} / 2)^{\text {inv }}$ and $\rho_{A}^{\text {inv }}(\xi)=\rho_{A}(\xi)=0$. Let $S$ be a connected component of $A(\mathbb{R}), j_{S}: S \hookrightarrow$ $A(\mathbb{R})$ the inclusion map, $x$ a point in $S$, and $q_{x}: A(\mathbb{R})_{0} \rightarrow S$ the restriction of $t_{x}(\mathbb{R})$. Note that $t_{x}(\mathbb{R}) \circ j=j_{S} \circ q_{x}$, where $j: A(\mathbb{R})_{0} \hookrightarrow A(\mathbb{R})$ is the inclusion map. Hence we have the following equalities in $H^{1}\left(A(\mathbb{R})_{0}, \mathbb{Z} / 2\right)$ :

$$
q_{x}^{*}\left(j_{S}^{*}(\xi)\right)=j^{*}\left(t_{x}(\mathbb{R})^{*}(\xi)\right)=j^{*}(\xi)=\rho_{A}(\xi)=0 .
$$

Since $q_{x}$ is a homeomorphism, $j_{S}^{*}(\xi)=0$. This in turn implies $\xi=0$ for $S$ is an arbitrary connected component of $A(\mathbb{R})$. Thus $\rho_{A}^{\text {inv }}$ is injective as asserted.

Now we prove $\mathrm{R}_{A}\left(H^{1}(A(\mathbb{C}), \mathbb{Z})\right) \subseteq H^{1}(A(\mathbb{R}), \mathbb{Z} / 2)^{\text {inv }}$. If $i: A(\mathbb{R}) \hookrightarrow A(\mathbb{C})$ is the inclusion map, then for any point $x$ in $A(\mathbb{R})$, we have $t_{x}(\mathbb{C}) \circ i=i \circ t_{x}(\mathbb{R})$, where $t_{x}(\mathbb{C}): A(\mathbb{C}) \rightarrow A(\mathbb{C})$ is the translation induced by $t_{x}$. Since $t_{x}(\mathbb{C})$ is homotopic to the identity map of $A(\mathbb{C})$,

$$
i^{*}=t_{x}(\mathbb{R})^{*} \circ i^{*}: H^{1}(A(\mathbb{C}), \mathbb{Z} / 2) \rightarrow H^{1}(A(\mathbb{R}), \mathbb{Z} / 2),
$$

which implies $i^{*}\left(H^{1}(A(\mathbb{C}), \mathbb{Z} / 2)\right) \subseteq H^{1}(A(\mathbb{R}), \mathbb{Z} / 2)^{\text {inv }}$. The inclusion under consideration follows at once.

It remains to prove $\rho_{A}\left(\mathrm{R}_{A}\left(H^{1}(A(\mathbb{C}), \mathbb{Z})\right)\right)=H^{1}\left(A(\mathbb{R})_{0}, \mathbb{Z} / 2\right)$. This however follows immediately since $r_{A}=\rho_{A} \circ \mathrm{R}_{A}$, the reduction modulo 2 homomorphism $\pi: H^{1}(A(\mathbb{C}), \mathbb{Z}) \rightarrow H^{1}(A(\mathbb{C}), \mathbb{Z} / 2)$ is surjective $(A(\mathbb{C})$ is a complex torus $)$, and there is a retraction $A(\mathbb{C}) \rightarrow A(\mathbb{R})_{0}$ (cf. [5, Theorem 3.1.5(ii)] for the last fact).

Recall that the number of connected components of $A(\mathbb{R})$ is $2^{c}$ for some integer $c$ satisfying $0 \leq c \leq \operatorname{dim} A$, cf. [5, Theorem 3.1.4]

Proposition 2.6. With notation as above,

$$
\begin{gathered}
R_{A}\left(H^{1}(A(\mathbb{C}), \mathbb{Z})^{G}\right)=\operatorname{Alg}^{1}(A(\mathbb{R})), \\
\operatorname{dim}_{\mathbb{Z} / 2} \operatorname{Alg}^{1}(A(\mathbb{R}))=c,
\end{gathered}
$$

where $2^{c}$ is the number of connected components of $A(\mathbb{R})$.

Proof. We have the following commutative diagram:

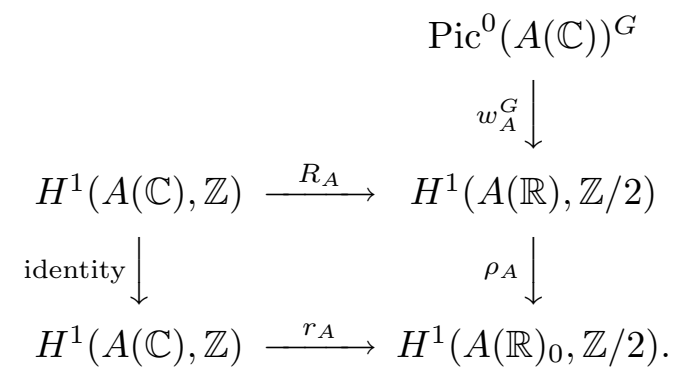


Therefore, in view of (2.3) and Lemmas 2.4 and 2.5, it is sufficient to prove the following two equalities:

(a) $r_{A}\left(H^{1}(A(\mathbb{C}), \mathbb{Z})^{G}\right)=\rho_{A}\left(w_{A}^{G}\left(\operatorname{Pic}^{0}(A(\mathbb{C}))^{G}\right)\right)$

(b) $\operatorname{dim}_{\mathbb{Z} / 2} r_{A}\left(H^{1}(A(\mathbb{C}), \mathbb{Z})^{G}\right)=c$.

We shall establish (a) and (b) by direct computation in terms of a suitable period matrix of $A$.

Set $n=\operatorname{dim} A$. Let $\Omega$ be a complex $n \times 2 n$ matrix such that the $\mathbb{Z}$-submodule $[\Omega]$ of $\mathbb{C}^{n}$ generated by the columns of $\Omega$ has rank $2 n$ and is mapped onto itself by the complex conjugation on $\mathbb{C}^{n} \rightarrow \mathbb{C}^{n}, z \rightarrow \bar{z}$. The complex conjugation on $\mathbb{C}^{n}$ gives rise to group action of $G$ on the complex torus $\mathbb{C}^{n} /[\Omega]$. If there exists a $G$-equivariant isomorphism between the complex Lie groups $\mathbb{C}^{n} /[\Omega]$ and $A(\mathbb{C})$, then $\Omega$ is said to be a period matrix of $A$. It is well known that $A$ admits a period matrix of the form

$$
\Omega=\left(I_{n}, \frac{1}{2} I_{n, k}+\sqrt{-1} S\right)
$$

where $I_{n}$ is the identity $n \times n$ matrix, $0 \leq k \leq n, I_{n, k}$ is obtained from $I_{n}$ by replacing columns $k+1$ through $n$ by the columns consisting of zeros, and $S$ is a real $n \times n$ matrix, cf. [5, Theorem 3.1.5].

Let $\Omega$ be as in (1), $\Lambda=[\Omega], T=\mathbb{C}^{n} / \Lambda$, and let $h: T \rightarrow A(\mathbb{C})$ be a $G$ equivariant isomorphism of complex Lie groups. The complex torus $T$ is endowed with an antiholomorphic involution $\sigma_{T}$ induced by the complex conjugation on $\mathbb{C}^{n}$. This allows us to use terminology and notation introduced at the beginning of this section. Denote by $T(\mathbb{R})_{0}$ the connected component of $T(\mathbb{R})=\{x \in$ $\left.T \mid \sigma_{T}(x)=x\right\}$ containing the identity element of the group $T(\mathbb{R})$. We have the following commutative diagram:

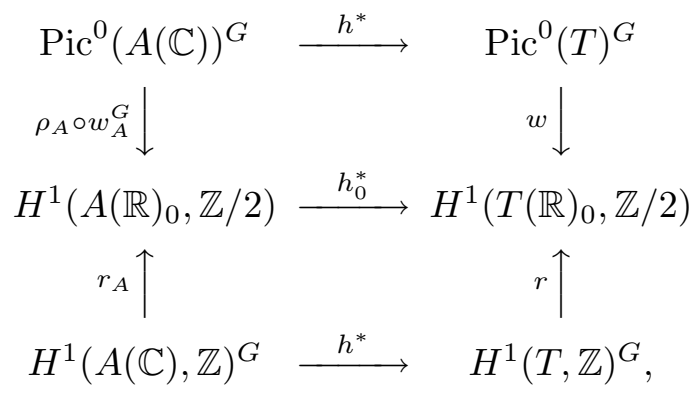

where $h_{0}: T(\mathbb{R})_{0} \rightarrow A(\mathbb{R})_{0}$ is the restriction of $h: T \rightarrow A(\mathbb{C}), w=h_{0}^{*} \circ \rho_{A} \circ w_{A}^{G} \circ$ $\left(h^{*}\right)^{-1}$, and $r: H^{1}(T, \mathbb{Z}) \rightarrow H^{1}\left(T(\mathbb{R})_{0}, \mathbb{Z} / 2\right)$ is the restriction homomorphism. It follows that (a) and (b) are equivalent to

$\left(a^{\prime}\right) \quad r\left(H^{1}(T, \mathbb{Z})^{G}\right)=w\left(\operatorname{Pic}^{0}(T)^{G}\right)$

$\left(b^{\prime}\right) \operatorname{dim}_{\mathbb{Z} / 2} r\left(H^{1}(T, \mathbb{Z})^{G}\right)=c$.

We shall now give a more direct description of the homomorphism $w: \operatorname{Pic}^{0}(T)^{G}$ $\rightarrow H^{1}\left(T(\mathbb{R})_{0}, \mathbb{Z} / 2\right)$. Given a holomorphic line bundle $L$ on $T$, we write $[L]$ for its isomorphism class. Each element of $\operatorname{Pic}^{0}(T)^{G}$ is of the form $[L]$ for some $L$ defined over $\mathbb{R}$, and in view of $(2.2)$,

$$
w([L])=w_{1}\left(L(\mathbb{R}) \mid T(\mathbb{R})_{0}\right) .
$$


Our next step is an explicit description of $\operatorname{Pic}^{0}(T)^{G}$ coming from the AppellHumbert theorem. We regard the unit circle $\mathbb{C}_{1}=\{v \in \mathbb{C}|| v \mid=1\}$ as a multiplicative group. For any $\chi$ in $\operatorname{Hom}\left(\Lambda, \mathbb{C}_{1}\right)$, define an action of $\Lambda$ on $\mathbb{C}^{n} \times \mathbb{C}$ by

$$
(\lambda,(z, v)) \rightarrow(z+\lambda, \chi(\lambda) v)
$$

for all $\lambda$ in $\Lambda$ and $(z, v)$ in $\mathbb{C}^{n} \times \mathbb{C}$. Denote by $L(\chi)$ the holomorphic line bundle on $T$ obtained by taking the quotient of $\mathbb{C}^{n} \times \mathbb{C}$ by $\Lambda$. Then

$$
P: \operatorname{Hom}\left(\Lambda, \mathbb{C}_{1}\right) \rightarrow \operatorname{Pic}^{0}(T), P(\chi)=[L(\chi)]
$$

is an isomorphism, cf. [16, p. 32, Theorem 2.3] or [18, p. 20]. There is a natural action of $G$ on $\operatorname{Hom}\left(\Lambda, \mathbb{C}_{1}\right), \chi \rightarrow \chi^{\sigma}$, where $\chi^{\sigma}(\lambda)=\overline{\chi(\bar{\lambda})}$ for all $\lambda$ in $\Lambda$. We have $\left[L\left(\chi^{\sigma}\right)\right]=[L(\chi)]^{\sigma}$ (cf. $[5$, Lemma 3.3.1]), and hence the isomorphism $P$ is $G$-equivariant.

Note that $T(\mathbb{R})_{0}$ can be described in a very explicit way as well. Indeed, regarding $\mathbb{R}^{n}$ as a subset of $\mathbb{C}^{n}$, we can also view $\mathbb{R}^{n} / \mathbb{Z}^{n}$ as a subtorus of $T=$ $\mathbb{C}^{n} / \Lambda(\Lambda=[\Omega]$ and $\Omega$ is as in (1)). Hence

$$
T(\mathbb{R})_{0}=\mathbb{R}^{n} / \mathbb{Z}^{n}
$$

The canonical isomorphisms $\Lambda \rightarrow H_{1}(T, \mathbb{Z})$ and $\mathbb{Z}^{n} \rightarrow H_{1}\left(T(\mathbb{R})_{0}, \mathbb{Z}\right)$ induce canonical isomorphisms

$$
\begin{aligned}
H_{\Lambda} & : H^{1}(T, \mathbb{Z})=\operatorname{Hom}\left(H_{1}(T, \mathbb{Z}), \mathbb{Z}\right) \rightarrow \operatorname{Hom}(\Lambda, \mathbb{Z}) \\
H & : H^{1}\left(T(\mathbb{R})_{0}, \mathbb{Z} / 2\right)=\operatorname{Hom}\left(H_{1}\left(T(\mathbb{R})_{0}, \mathbb{Z}\right), \mathbb{Z} / 2\right) \rightarrow \operatorname{Hom}\left(\mathbb{Z}^{n}, \mathbb{Z} / 2\right) .
\end{aligned}
$$

Defining an action of $G$ on $\operatorname{Hom}(\Lambda, \mathbb{Z})$ by $\varphi \rightarrow \varphi^{\sigma}$, where $\varphi$ is in $\operatorname{Hom}(\Lambda, \mathbb{Z})$ and $\varphi^{\sigma}(\lambda)=\varphi(\bar{\lambda})$ for all $\lambda$ in $\Lambda$, we see that the isomorphism $H_{\Lambda}$ is $G$-equivariant.

We shall now find connections between the isomorphisms $P, H_{\Lambda}, H$, which will reduce $\left(\mathrm{a}^{\prime}\right)$ and $\left(\mathrm{b}^{\prime}\right)$ to simple algebraic statements.

If $\chi$ is in $\operatorname{Hom}\left(\Lambda, \mathbb{C}_{1}\right)^{G}$, then $\chi(\Lambda) \subseteq\{-1,1\}$, and hence we can define a homomorphism

$$
\omega: \operatorname{Hom}\left(\Lambda, \mathbb{C}_{1}\right)^{G} \rightarrow \operatorname{Hom}\left(\mathbb{Z}^{n}, \mathbb{Z} / 2\right)
$$

by $\omega(\chi)(\nu)=e(\chi(\nu))$ for all $\nu$ in $\mathbb{Z}^{n}$, where $e:\{-1,1\} \rightarrow \mathbb{Z} / 2$ is the unique group isomorphism.

Define a homomorphism

$$
\pi: \operatorname{Hom}(\Lambda, \mathbb{Z}) \rightarrow \operatorname{Hom}\left(\mathbb{Z}^{n}, \mathbb{Z} / 2\right)
$$

by $\pi(\varphi)(\nu)=\varphi(\nu)(\bmod 2)$ for all $\varphi$ in $\operatorname{Hom}(\Lambda, \mathbb{Z})$ and $\nu$ in $\mathbb{Z}^{n}$.

We claim that the following diagram is commutative: 


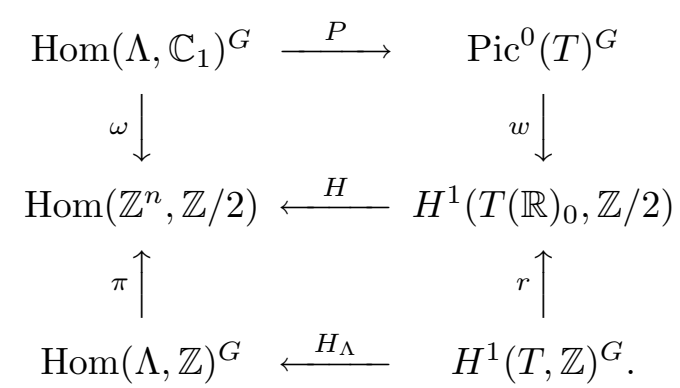

Since $P$ and $H_{\Lambda}$ are $G$-equivariant isomorphisms, the upper and lower horizontal arrows are well defined isomorphisms. It is clear that the lower square is commutative. In order to show that the upper square is commutative we need an auxiliary construction.

Given an element $\alpha$ in $\operatorname{Hom}\left(\mathbb{Z}^{n}, \mathbb{Z} / 2\right)$, define an action of $\mathbb{Z}^{n}$ on $\mathbb{R}^{n} \times \mathbb{R}$ by

$$
(\nu,(x, u)) \rightarrow\left(x+\nu,(-1)^{\alpha(\nu)} u\right)
$$

for all $\nu$ in $\mathbb{Z}^{n}$ and $(x, u)$ in $\mathbb{R}^{n} \times \mathbb{R}$. Let $M(\alpha)$ be the real line bundle on $T(\mathbb{R})_{0}=\mathbb{R}^{n} / \mathbb{Z}^{n}$ obtained by taking the quotient of $\mathbb{R}^{n} \times \mathbb{R}$ by $\mathbb{Z}^{n}$. We assert

$$
H\left(w_{1}(M(\alpha))\right)=\alpha .
$$

If $n=1$, then (4) follows immediately. Consequently, we readily get $H\left(w_{1}\left(M\left(\alpha_{i}\right)\right)\right)$ $=\alpha_{i}$ for $1 \leq i \leq n$, where $\alpha_{i}(\nu)=\nu_{i}(\bmod 2)$ and $\nu_{i}$ is the $i$ th component of $\nu$ in $\mathbb{Z}^{n}$ (think of $M\left(\alpha_{i}\right)$ as a pullback of a real line bundle on $\mathbb{R} / \mathbb{Z}$ ). Since the line bundles $M(\alpha+\beta)$ and $M(\alpha) \otimes M(\beta)$ are isomorphic for all $\alpha$ and $\beta$ in $\operatorname{Hom}\left(\mathbb{Z}^{n}, \mathbb{Z} / 2\right)$, we conclude that $(4)$ holds.

By construction, if $\chi$ is in $\operatorname{Hom}\left(\Lambda, \mathbb{C}_{1}\right)^{G}$, then $L(\alpha)(\mathbb{R}) \mid T(\mathbb{R})_{0}$ is isomorphic to $M(\omega(\chi))$. Hence the upper square of $(3)$ is commutative in view of $(2),(4)$, and the definition of $P$.

Commutativity of $(3)$ implies that $\left(\mathrm{a}^{\prime}\right)$ and $\left(\mathrm{b}^{\prime}\right)$ are equivalent to

$\left(a^{\prime \prime}\right) \omega\left(\operatorname{Hom}\left(\Lambda, \mathbb{C}_{1}\right)^{G}\right)=\pi\left(\operatorname{Hom}(\Lambda, \mathbb{Z})^{G}\right)$

$\left(b^{\prime \prime}\right) \quad \operatorname{dim}_{\mathbb{Z} / 2} \pi\left(\operatorname{Hom}(\Lambda, \mathbb{Z})^{G}\right)=c$.

Recall that $\Lambda=[\Omega]$, where $\Omega=\left(I_{n}, \frac{1}{2} I_{n, k}+\sqrt{-1} S\right)$ is as in (1). Let $\epsilon_{1}, \ldots, \epsilon_{n}$ (resp. $\left.\gamma_{1}, \ldots, \gamma_{n}\right)$ be the columns of $I_{n}$ (resp. $S$ ). Setting $\tau_{j}=\frac{1}{2}\left(\epsilon_{j}+\gamma_{j}\right)$ for $1 \leq j \leq k$, we obtain a $\mathbb{Z}$-basis

$$
\left(\epsilon_{1}, \ldots, \epsilon_{n}, \tau_{1}, \ldots, \tau_{k}, \gamma_{k+1}, \ldots, \gamma_{n}\right)
$$

for the lattice $\Lambda$ satisfying

$$
\bar{\tau}_{j}=\epsilon_{j}-\tau_{j} \text { for } 1 \leq j \leq k .
$$

Obviously, $\left(\epsilon_{1}, \ldots, \epsilon_{n}\right)$ is the standard $\mathbb{Z}$-basis for $\mathbb{Z}^{n}$. Setting

$$
\Gamma=\left\{\alpha \in \operatorname{Hom}\left(\mathbb{Z}^{n}, \mathbb{Z} / 2\right) \mid \alpha\left(\epsilon_{j}\right)=0 \text { for } 1 \leq j \leq k\right\},
$$

we assert

$$
\pi\left(\operatorname{Hom}(\Lambda, \mathbb{Z})^{G}\right)=\Gamma=\omega\left(\operatorname{Hom}\left(\Lambda, \mathbb{C}_{1}\right)^{G}\right) .
$$


In order to prove (7) consider the following commutative diagram:

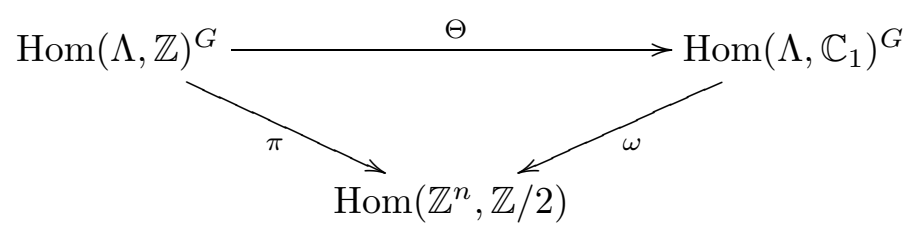

where $\Theta(\varphi)(\lambda)=(-1)^{\varphi(\lambda)}$ for all $\varphi$ in $\operatorname{Hom}(\Lambda, \mathbb{Z})^{G}$ and $\lambda$ in $\Lambda$. In particular, we get

$$
\pi\left(\operatorname{Hom}(\Lambda, \mathbb{Z})^{G}\right) \subseteq \omega\left(\operatorname{Hom}\left(\Lambda, \mathbb{C}_{1}\right)^{G}\right) .
$$

Since $(5)$ is a basis for $\Lambda$, the inclusion $\Gamma \subseteq \pi\left(\operatorname{Hom}(\Lambda, \mathbb{Z})^{G}\right)$ is obvious. Thus to complete the proof of (7) it remains to show

$$
\rho\left(\operatorname{Hom}\left(\Lambda, \mathbb{C}_{1}\right)^{G}\right) \subseteq \Gamma .
$$

If $\chi$ is in $\operatorname{Hom}\left(\Lambda, \mathbb{C}_{1}\right)^{G}$, then $\chi(\Lambda) \subseteq\{-1,1\}$, and hence, in view of (6), for each $j$ satisfying $1 \leq j \leq k$, we have

$$
\chi\left(\tau_{j}\right)=\chi\left(\bar{\tau}_{j}\right)=\chi\left(\epsilon_{j}-\tau_{j}\right)=\chi\left(\epsilon_{j}\right) \chi\left(\tau_{j}\right)^{-1}=\chi\left(\epsilon_{j}\right) \chi\left(\tau_{j}\right),
$$

which implies $\chi\left(\epsilon_{j}\right)=1$. Hence $\omega(\chi)$ belongs to $\Gamma$ and the inclusion under consideration is proved. The proof of $(7)$ is now complete.

Note that $\operatorname{dim}_{\mathbb{Z} / 2} \Gamma=n-k$. Since (5) is a $\mathbb{Z}$-basis for $\Lambda$, it follows that $T(\mathbb{R})$ has $2^{n-k}$ connected components, cf. [5, Theorems 3.1.4, 3.1.5]. Thus $A(\mathbb{R})$ also has $2^{n-k}$ connected components, and hence $c=n-k$. Combining this with (7), we conclude that $\left(\mathrm{a}^{\prime \prime}\right)$ and $\left(\mathrm{b}^{\prime \prime}\right)$ are satisfied. The proposition is proved.

Proof of Theorem 1.1. Step 1. Assume that $X=A$ is an Abelian variety. With notation as in Section 1 and Proposition 2.6,

$$
i_{A}^{2}\left(H^{2}\left(G, H^{1}(A(\mathbb{C}), \mathbb{Z})\right)\right)=\mathrm{R}_{A}\left(H^{1}(A(\mathbb{C}), \mathbb{Z})^{G}\right)=\operatorname{Alg}^{1}(A(\mathbb{R})) .
$$

Furthermore, $\operatorname{dim}_{\mathbb{Z} / 2} \operatorname{Alg}^{1}(A(\mathbb{R}))=c$, where $2^{c}$ is the number of connected components of $A(\mathbb{R})$. On the other hand, it is also known that

$$
\operatorname{dim}_{\mathbb{Z} / 2} H^{2}\left(G, H^{1}(A(\mathbb{C}), \mathbb{Z})\right)=c,
$$

(cf. [5, proof of Theorem 2.7.9], note that $H^{2}\left(G, H^{1}(A(\mathbb{C}), \mathbb{Z})\right)=$ $H^{1}\left(G, H^{1}(A(\mathbb{C}), \mathbb{Z}(-1))\right)$, where the latter group is introduced in [5]). Hence $i_{A}^{2}$ is injective.

Step 2. We shall now deal with arbitrary $X$. Let $A$ be the Albanese variety of $X$ and let $\alpha: X \rightarrow A$ be the Albanese morphism corresponding to some point in $X(\mathbb{R})$. In particular, $A$ and $\alpha$ are defined over $\mathbb{R}$. Let $\alpha(\mathbb{C}): X(\mathbb{C}) \rightarrow A(\mathbb{C})$ and $\alpha(\mathbb{R}): X(\mathbb{R}) \rightarrow A(\mathbb{R})$ be the maps determined by $\alpha$. It is well known that

$$
\alpha(\mathbb{C})^{*}: H^{1}(A(\mathbb{C}), \mathbb{Z}) \rightarrow H^{1}(X(\mathbb{C}), \mathbb{Z})
$$

is an isomorphism. Since $\alpha(\mathbb{C})^{*}$ is $G$-equivariant, it induces an isomorphism

$$
\alpha(\mathbb{C})^{2}: H^{2}\left(G, H^{1}(A(\mathbb{C}), \mathbb{Z})\right) \rightarrow H^{2}\left(G, H^{1}(X(\mathbb{C}), \mathbb{Z})\right),
$$


included in the following commutative diagram:

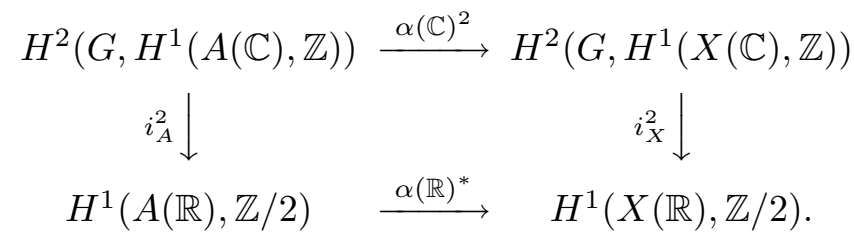

As observed in $\left[13\right.$, Lemma 2.1], $\alpha(\mathbb{R})^{*}\left(\operatorname{Alg}^{1}(A(\mathbb{R}))\right)=\operatorname{Alg}^{1}(X(\mathbb{R}))$, and hence Step 1 implies

$$
i_{X}^{2}\left(H^{2}\left(G, H^{1}(X(\mathbb{C}), \mathbb{Z})\right)\right)=\operatorname{Alg}^{1}(X(\mathbb{R})) .
$$

The proof is complete.

\section{References}

[1] M. Abánades and W. Kucharz, Algebraic equivalence of real algebraic cycles, Ann. Inst. Fourier (Grenoble) 49, (1999), 1797-1804.

[2] J. Bochnak, M. Coste and M.-F. Roy, Real Algebraic Geometry, Ergebnisse der Mathematik und ihrer Grenzgebiete, 36, Berlin Heidelberg, Springer, 1998.

[3] J. Bochnak and W. Kucharz, On homology classes represented by real algebraic varieties, Singularities Symposium - Lojasiewicz 70, Banach Center Publ. 44, 21-35, Warszawa, 1998.

[4] A. Borel et A. Haefliger, La classe d'homologie fondamentale d'un espace analytique, Bull. Soc. Math. France, 89 (1961), 461-513.

[5] C. Ciliberto and C. Pedrini, Real abelian varieties and real algebraic curves, Lectures in real geometry (Madrid, 1994), 167-256, de Gruyter Exp. Math., 23, de Gruyter, Berlin, 1996.

[6] A. Comessatti, Sulle varietá abeliane reali I e II, Ann. Mat. Pura e Appl. 2-4 (1924-1926), 67-106 e 27-71.

[7] W. Fulton, Intersection Theory, Ergebnisse der Mathematik und ihrer Grenzgebiete, 2, Berlin Heidelberg New York, Springer, 1984.

[8] P. Griffiths and J. Harris, Principles of Algebraic Geometry, Wiley-Interscience, 1978.

[9] B. Gross and J. Harris Real algebraic curves, Ann. Sci. Éc. Norm Sup. Paris 14(1981), 157-182.

[10] A. Grothendieck, Technique de descente et théorèmes d'existence en géométrie algebrique, I - IV, Séminaire Bourbaki, Éposés 190, 195, 212, 221, 232, 236, 1959-62.

[11] J. Huisman, Real abelian varieties with complex multiplication, Ph.D. thesis, Free University of Amsterdam, 1992.

[12] W. Kucharz, Algebraic equivalence and homology classes of real algebraic cycles, Math. Nachr. 180 (1996), 135-140.

[13] W. Kucharz, Algebraic equivalence of real divisors, Math. Zeit. 238 (2001), 817-827.

[14] W. Kucharz, Algebraic cycles and algebraic models of smooth manifolds, J. Algebraic Geometry 11(2002), 101-127.

[15] W. Kucharz, Algebraic equivalence of cycles and algebraic models of smooth manifolds, Compo. Math., to appear.

[16] H. Lange and Ch. Birkenhake, Complex Abelian Varieties, Grundlehren der mathematischen Wissenschaften, 302, Berlin Heidelberg, Springer, 1992.

[17] J. S. Milne, Abelian varieties, Arithmetic Geometry, 103-150, Berlin Heidelberg New York, Springer, 1986.

[18] D. Mumford, Abelian Varieties, Oxford Univ. Press, Oxford, 1970.

[19] J. P. Serre, Géométrie algébrique et géométrie analytique, Ann. Inst. Fourier (Grenoble) 6 (1956), 1-42. 
[20] R. Silhol, Real Algebraic Surfaces, Lecture Notes in Math. 1392, Springer, 1989.

Department of Mathematics and Statistics, University of New Mexico, AlbuQUERQue, New MeXiCO 87131-1141, U.S.A.

E-mail address: kucharz@math.unm.edu 